\title{
Algunos predictores de la conducta prosocial-altruista en la infancia: empatía, toma de perspectiva, apego, modelos parentales, disciplina familiar e imagen del ser humano
}

\author{
M. J. Otiz, P. Apodaka, I. Etxeberria, A. Ezeiza, \\ M. J. Fuentes y F. López \\ U.P.V., Universidad de Málaga, Universidad de Salamanca
}

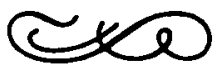

\begin{abstract}
Resumen
La presente investigación se realizó para a) analizar la capacidad predictora de las siguientes variables en la conducta prosocial altruista: la empatía disposicional y situacional, la toma de perspectiva, la imagen del ser bumano, la seguridad del apego, el modelo de conducta prosocial de los padres y el tipo de disciplina utilizada más frecuentemente por los progenitores, $y$ b) establecer las variables más discriminantes entre los grupos de sujetos más extremos en el continuo altruismo-no altruismo. Muestra: El estudio se realizó con 165 sujetos de ambos sexos, de edades compredidas entre los 10 y los 12 años, en diversos centros de Salamanca y San Sebastián. Resultados: Todos los factores estudiados, salvo el mole taking, mostraron su capacidad predictiva en el comportamiento altruista prosocial, siendo los más discriminantes los siguientes: la empatía disposicional, la seguridad del apego, la empatía situacional centrada en la víctima y la disciplina inductiva.
\end{abstract}

Palabras clave: altruismo, conducta prosocial, empatía, apego, role taking.

\section{Some predictors of prosocial behavior (altruism) in children}

\begin{abstract}
The purpose of our study was a) to analyze the predictive role of the following variables in prosocialaltruistic behaviour: empathy (situational and dispositional), role-taking, the representation of buman nature, security of attachment, the parental model of prosocial behaviour, and parental discipline techniques, and b) to establish between these variables which are the ones that best discriminate the most and least altruistic subjects. Sample. 165 children, aged 10-12 yrs., form San Sebastián and Salamanca participated in this study. Results: All the variables studied, except role-taking, showed significant predictive power in prosocial-altruistic behaviour, the most discriminant ones being: dispositional empathy, security of attachment, situational empatby, and the induction as discipline technique in the family context.
\end{abstract}

Key words: Altruism, prosocial behavior, empathy, attachment, rol-taking.

Dirección del autor: Dpto. de Psicología. Facultad de Psicología. Aptdo. 1249. San Sebastián.

Original recibido: Diciembre, 1990. 


\section{INTRODUCCION}

El trabajo que aquí presentamos se enmarca en un proyecto de investigación más amplio que intenta responder progresivamente a una serie de cuestiones todavía pendientes en el estudio de la conduca prosocial y altruista, como son: la misma definición de esta conducta, sus predictores afectivos, cognitivos y situacionales, las formas de medida, las posibilidades educativas para desarrollarla, etc. Parte de estas cuestiones se han tratado en estudios previos (Fuentes y otros, en prensa). En el primer abordaje del tema el esfuerzo se dirigió a la definición conceptual de la conducta prosocial-altruista y al estudio de la capacidad predictiva de algunos de los factores asociados a ella.

Respecto a la primera de estas cuestiones, tras reflexionar sobre las distintas opciones (Macaulay y Berkowitz, 1970; Staub, 1979; Kalliopuska, 1980; Eisenberg, 1982; Chacon, 1986) y problemas en torno a la conceptualización de este comportamiento, desde un principio se optó por estudiar la conducta prosocial que cumple los requisitos externos de la conducta altruista, a la que llamamos conducta «prosocial-altruista». Estos criterios son los siguientes:

1. Beneficiar a otra persona.

2. Ser voluntaria.

3. Que esté bien definido el beneficiario de la acción, de modo que no se considera el hecho de que terceras personas obtengan beneficios.

4. Que quien ejecuta la acción no pueda anticipar beneficios externos para sí, asociados a su conducta.

Respecto a los factores que predicen este comportamiento prosocial-altruista, en el estudio piloto se analizaron los factores, tanto cognitivos como afectivos, de carácter más estable, ya que en último término esta investigación pretendía servir de fundamento teórico para una intervención educativa posterior.

En concreto, en esa primera investigación se estudió la capacidad predictiva de la empatía disposicional y situacional, la toma de perspectiva y la imagen que tienen los niños de la naturaleza humana en la conducta prosocial-altruista.

En el trabajo que presentamos aquí nos proponemos analizar la influencia de las siguientes variables sobre la conducta prosocial-altruista.

La empatia ha sido definida por Hoffman $(1975,1981,1982,1983)$ como la experiencia afectiva vicaria de los sentimientos de otra persona. Se considera el resultado de un proceso interactivo entre componentes cognitivos y afectivos que van evolucionando a medida que avanza el desarrollo. En la abundante literatura sobre el tema se suele distinguir entre empatía disposicional o rasgo y empatía situacional o estado. La empatía disposicional consiste en una tendencia relativamente estable de la persona a percibir y experimentar de forma vicaria los afectos de otras personas, mientras que por empatía situacional se entiende el grado de experiencia afectiva vicaria que tienen las personas en una situación concreta.

La mayoría de los estudios han considerado uno u otro tipo de empatía en relación a las conductas altruistas (Batson y otros, 1978; Batson y Coke, 1981; Fultz y otros, 1986). Estos trabajos han mostrado una consistente relación entre la empatía (disposicional o situacional) y la conducta prosocial-altruista, especialmente entre sujetos adultos. En el caso de los niños, los datos de la literatura empírica son menos concordantes respecto a la empatía rasgo; sin embargo, en nuestro anterior trabajo sí hallamos, en una muestra de niños, una correlación significativa entre empatía disposicional y altruismo. 
Otra importante variable predictiva es la toma de perspectiva o capacidad para ponerse en el lugar del otro, comprender sus pensamientos, sentimientos, motivos y conductas. Algunos autores (Iannotti, 1978, 1985) han relacionado esta capacidad con la activación empática y con la realización de conductas prosociales, pero no existe un acuerdo unánime entre los numerosos estudios realizados sobre este tema. En un intento por clarificar el estado de la cuestión, Underwood y More (1982) llevaron a cabo, a través de un meta-análisis, una amplia revisión de la literatura existente. Los resultados de su trabajo mostraron que la correlaciones significativas se daban en los estudios realizados con adultos, mientras que en las investigaciones con niños y adolescentes la consistencia de los resultados era escasa. Sin embargo, en nuestro estudio inicial también se encontró una relación significativa entre ambas variables en una muestra de niños de 10 a 12 años.

Imagen del ser humano. Krebs y otros (1985) señalan que las personas que tienen un concepto más positivo del ser humano y de lo que se puede esperar de las relaciones interpersonales, tienen mayor disponibilidad para realizar conductas prosociales y altruistas que quienes creen que las personas buscan siempre su propio interés.

La importancia de la historia afectiva en la conducta social en general y en la conducta altruista en particular ha sido reiteradamente demostrada por la literatura empírica. Por nuestra parte, pretendemos analizar el poder predictivo de la calidad del apego en la conducta prosocial altruista. Creemos que existen suficientes y coherentes razones para predecir una estrecha relación entre la seguridad del apego y el comportamiento prosocial-altruista.

En primer lugar, desde una perspectiva etológica, la especie humana, como otras, a lo largo de la evolución ha desarrolado aquellos motivos y recursos de conducta necesarios para la supervivencia, entre los que se encuentran las predisposiciones a actuar de manera altruista en situaciones de necesidad de los miembros de la familia o del grupo. Por ello, el marco de las relaciones de apego constituye un excelente contexto donde los niños pueden desarrollar los intercambios y sentimientos afectivos sobre los que se asienta la empatía, y donde aprender las estrategias y situaciones de respuesta altruista.

En este sentido, y continuando con el contexto familiar, no cabe duda de que el comportamiento prosocial de los progenitores constituirá una variable predictora de gran poder en el desarrollo de la conducta prosocial altruista, pues los progenitores, por su investimento emocional, son los modelos de imitación más eficaces para los niños (Hoffman, 1975; Bandura, 1987).

Por último, consideramos necesario abordar una variable que tuvo que ser eliminada en el estudio previo debido a la mortalidad de la muestra, nos referimos al tipo de disciplina utilizada por los padres para controlar y/o corregir la conducta no deseable en los niños. Aunque los padres no utilizan un único tipo de disciplina, de acuerdo con Hoffman (1970, 1977, 1980, 1983), se puede evaluar la opción más frecuente en una de estas tres grandes categorías: afirmación de poder, inducción y retirada de amor. Según los estudios realizados por este autor y los trabajos de réplica posteriores, la afirmación de poder y la retirada de amor no predicen comportamientos de ayuda o altruistas, y además la segunda genera ansiedad, mientras que la inducción, al favorecer la capacidad de toma de perspectiva y la empatía con la víctima, y al desarrollar la naturaleza conceptual del conflicto, muestra un importante peso como factor predictor del comportamiento prosocial-altruista. 
La presente investigación, en un intento de avanzar en la línea abierta por el trabajo previo, se diseñó para afrontar los siguientes objetivos:

a) Corroborar los resultados obtenidos anteriormente en torno a la capacidad predictora de la empatía disposicional, la empatía situacional, la toma de perspectiva y la imagen del ser humano.

b) Analizar el poder predictivo de otras variables señaladas por la literatura como relevantes: la seguridad del apego, el modelo de conducta prosocial de los padres y el tipo de displina utilizada más frecuentemente por los progenitores.

c) Establecer las variables más discriminantes entre los grupos de sujetos más extremos en el continuo altruismo-no altruismo.

\section{HIPOTESIS}

Las hipótesis que nos planteamos fueron las siguientes:

1. Se predijo una asociación significativa y positiva entre la empatía disposicional, la empatía situacional, la capacidad de reconocer la perspectiva del otro, la concepción positiva del ser humano, la seguridad del apego, la disciplina inductiva y el modelo de comportamiento prosocial de los progenitores con la conducta prosical altruista de los niños, lo que calificaría a dichas variables de predictores.

2. La empatía disposicional, la empatía situacional, la capacidad de reconocer la perspectiva del otro, la concepción positiva del ser humano, la seguridad del apego, la disciplina inductiva y el modelo de comportamiento prosocial de los progenitores configurarían el perfil característico de los sujetos clasificados como altruistas, con valores significativamente superiores a los mostrados por el grupo seleccionado como no altruista.

\section{MATERIAL Y METODO}

\subsection{Sujetos}

El estudio se realizó con 165 sujetos de ambos sexos, de edades comprendidas entre los 10 y los 12 años, que cursaban $5 .^{\circ}$ y $6 .^{\circ}$ curso de E.G.B. en diversos centros de Salamanca y San Sebastián (ver Cuadro I).

\section{CUADRo I}

Relación de centros y sujetos de la muestra

\begin{tabular}{|llccc|}
\hline CIUDAD & TIPO CENTRO & GRUPO & VARONES & MUJERES \\
\hline Salamanca & Público & 2 & 27 & 23 \\
San Sebastián & Privado & 1 & - & 45 \\
San Sebastián & Ikastola & 2 & 33 & 37 \\
\hline
\end{tabular}




\subsection{Instrumentos de medida}

\subsubsection{Medidas de la variable criterio «Conducta prosocial-altruista»}

Se utilizaron cuatro tipos diferentes de indicadores de la conducta prosocialaltruista:

a) En primer lugar, después de plantear a los niños una situación en la que otros niños necesitaban ayuda debido a que habían sufrido unas inundaciones, se les pasó un cuestionario que recogía diferentes tipos de ayuda para que señalaran cuáles estaban dispuestos a ofrecer: «Cuestionario de ayudas que prometo hacer» o «Ayuda informada». Las opciones de ayuda que se presentaban eran las siguientes:

- Dar dinero: 0,5, 25, 50 o 100 pesetas.

- Dar juguetes, cuentos o libros: $0,1,2,3$ o 4 .

- Trabajar durante el recreo: 0, 1, 2 o 3 días.

b) A continuación se contabilizó la cantidad de dinero, el número de juguetes, cuentos o libros, y si dedicaron o no su tiempo libre para trabajar a favor de los niños de Colombia: «Ayuda real».

c) Además, se realizó un «Cuestionario Sociométrico» en el que, en primer lugar, a cada niño se le pedía que nombrara: a) los tres niños de su clase que más ayudan normalmente y b) los tres más egoístas. En segundo lugar, se presentaban a cada niño, en diferentes días, dos listas de todos los compañeros de clase para que evaluara (de 1 a 5) el grado en que cada compañero 1) ayuda a los demás en tareas escolares, y 2) consuela o defiende a los demás cuando lo necesitan.

d) Por último, se pidió a los maestros que evaluaran a todos sus alumnos de 1 a 5 en cada una de las tres categorías ya mencionadas: «conducta de defensa y consuelo», y «ayuda escolar».

\subsubsection{Medidas de los factores predictores}

«La capacidad de toma de perspectiva social» de los sujetos se midió con una adaptación de la prueba de congnición social elaborada por Zahn-Waxler, Iannotti y Chapman (1982). Esta prueba evalúa la capacidad del niño para captar diversos tipos de conductas en los demás (respuestas empáticas, de intervención social, de culpa, de autopreocupación y agresivas), y en general, la situación en la que se encuentran los otros. Aquí se utilizaron cinco historias, graduadas en orden de menor a mayor complejidad de las emociones y los pensamientos implicados.

«La empatía disposicional» o empatía rasgo se midió a través del cuestionario de Empatía de Merhabian y Epstein (1972).

«La empatía situacional» se midió utilizando una adaptación del «Autoinforme de respuesta emocional» de Toi y Batson (1982), que incluía ítems relacionados con la empatía centrada en sí mismo, empatía centrada en la víctima, percepción social adecuada e inadecuada.

Para evaluar «la imagen del ser bumano» que tienen los niños se utilizó un diferencial semántico sobre el grado de bondad, maldad, generosidad, egoísmo, simpatía, cariño y alegría de la gente.

La medida del «tipo de disciplina» más frecuentemente utilizada por los pa- 
dres fue una adaptación de la prueba de Hoffman y Salztein (1967), en la que cada progenitor, ante cuatro hipotéticas faltas cometidas por su hijo/a, señalaba en un lista de opciones propuestas la primera, segunda y tercera respuestas más frecuentes ante cada situación. Esta lista contenía nueve respuestas posibles, tres por cada una de la categorías de disciplina.

Para evaluar «el grado de focalización en la víctima de la disciplina parental» se adaptó la prueba utilizada por Hoffman (1975). En ella, niños y progenitores expresaban la técnica disciplinaria más frecuentemente utilizada por el padre y por la madre (respuestas abiertas) ante tres situaciones en las que el niño provocaba un daño a otra persona, a) con provocación por parte de ésta, b) sin provocación, pero con apoyo de un compañero, y c) sin provocación, ni apoyo social.

La variable «modelo de conducta prosocial-altruista de los progenitores» se evaluó a través de un cuestionario en el que los niños contestaban si habían visto a su padre y a su madre realizar una serie de conductas prosociales-altruistas. Las opciones de respuesta se graduaban desde «nunca» a «muchas veces».

Para evaluar «la seguridad del apego» se elaboró un cuestionario en el que los niños respondían a ítems relativos al grado de responsividad, protección, intimidad, afecto y seguridad aportada por la madre y el padre.

\subsubsection{Procedimiento}

Para llevar a cabo este estudio se realizaron cinco sesiones de contacto con los niños en cada centro, cuyo contenido y objetivos pasamos a describir.

En una primera sesión, dos personas, un varón y una mujer, que fueron presentados por los maestros como miembros de una asociación internacional de ayuda, hablaron a los niños de la situación que estaban padeciendo los niños de Colombia después de las inundaciones. Tras esto, repartieron a cada niño una hoja para que señalaran en ella el tipo de ayuda que estaban dispuestos a ofrecer («Cuestionario de ayudas que prometo hacer»). Se recalcaba que la ayuda era voluntaria y que si querían dar algo debía ser algo propio (su dinero, sus juguetes o su tiempo de recreo).

Entre el relato de la situación y el reparto de las hojas de respuesta, se pasó a los niños el «Cuestionario de empatía situacional».

Tras recoger los cuestionarios de "Ayudas que prometo hacer», se dijo a los niños que los que quisieran se podían quedar en la clase, durante el tiempo de recreo, para ayudar a enviar unas cosas a los niños de Colombia. Se controló quiénes se quedaron en la clase y quiénes no. A los niños que se quedaron se les pidió que escribieran una carta o realizaran un dibujo para enviárselo a los niños de Colombia en un sobre, donde debían poner el remite con su nombre y dirección.

Una semana más tarse se volvió al colegio para recoger el dinero y los juguetes que habían llevado los niños.

En la segunda sesión otras personas, que aparecían como totalmente independientes de la pareja anterior, pasaron a los niños los cuestionarios de «imagen del ser humano» y «empatía disposicional», y a los niños y tutores la primera parte del sociograma, referida a «ayuda a tareas escolares».

En otra visita posterior, las mismas personas pasaron a niños y maestros la segunda parte del sociograma (referida a conductas de consuelo y defensa), y a los alumnos, además, el cuestionario de apego y el de imagen de conducta prosocial de los padres. 
La cuarta sesión, realizada una semana más tarde, se dedicó a pasar a niños y tutores el segundo sociograma, el cuestionario de apego y el de modelo de conducta prosocial de los padres. Asimismo, en esta sesión se repartieron entre los niños los sobres en los que se enviaba a los padres los cuestionarios sobre disciplina.

El siguiente paso, tras esta primera fase, fue la selección de los grupos más y menos altruistas de cada clase. A las hora de asignar los sujetos a los grupos alto y bajo en altruismo, se analizaron las puntuaciones de los sujetos en los distintos índices de "conducta prosocial-altruista», tomando como criterio la constancia en las divesas pruebas (ayuda informada, ayuda real, eleciones compañeros, sociograma compañeros y sociograma tutores), y no simplemente la puntuación alta o baja en un determinado índice. De este modo se eligió a los niños que más y que menos puntuaciones lograron en las medidas de «conducta prosocial-altruista» dentro de cada grupo de clase.

En la quinta y última sesión se pasó a todos los niños el cuestionario sobre disciplina de los padres y a los niños seleccionados la prueba individual de toma de perspectiva.

\subsection{Tratamiento de datos}

Para responder a los interrogantes planteados en este trabajo se realizaron fundamentalmente dos tipos de análisis estadísticos.

El primer tipo lo constituyen correlaciones de Pearson entre las variables predictoras y las medidas de conducta prosocial-altruista y el total de conducta prosocial-altruista. Con ello pretendíamos poner a prueba las hipótesis que habíamos planteado anteriormente intentando ver en qué medida está asociada cada variable con la conducta prosocial. Estos análisis se llevaron a cabo tomando como muestra todos los sujetos.

El segundo tipo de análisis pretende estudiar la potencia predictiva y explicativa del conjunto de factores seleccionados por nosotros como más relevantes, y describir con ellos el perfil discriminante caracterítico de los sujetos altos y bajos de conducta altruista. Para ello se contó solamente con los sujetos clasificados como altos y bajos en altruismo, 16 y 14, respectivamente.

\section{RESULTADOS}

Comenzaremos por presentar las correlaciones obtenidas entre las variables predictoras y la medida total de la conducta prosocial-altruista en la muestra total, y en las mujeres y los varones por separado (Hipótesis 1).

Empatía y conducta prosocial-altruista. Como puede observarse en la Tabla 1, la empatía como rasgo de personalidad o empatía disposicional no mostró una correlación significativa con la conducta prosocial-altruista. Sin embargo, tal como se predecía, la experiencia afectiva empática con la víctima en una determinada situación sí se reveló como una variable con poder predictivo de la conducta prosocial altruista, siendo ligreramente más fuerte la asociación en las mujeres. En este sentido, nuestros resultados, al menos en la muestra de todos los sujetos, corroboran la literatura empírica, que muestra resultados concluyentes respecto al valor predictivo de la empatía situacional, mientras que los datos relativos a la relación entre empatía disposicional y comportamiento prosocial se revelan más inconsistentes. 
Asimismo, también corroborando nuestras predicciones, la empatía situacional centrada en sí mismo o los sentimientos de ansiedad y malestar en nuestro estudio no mostró una asociación significativa con la conducta prosocialaltruista.

TABLA 1

Empatía y conducta prosocial-altruista

\begin{tabular}{lccc}
\hline & \multicolumn{3}{c}{ Conducta prosocial-altruista } \\
& Muestra total & Mujeres & Varones \\
Empatía disposicional & .0525 & .0229 & .0439 \\
Empatía situacional centrada en la & & & .2784 \\
víctima & $.2623^{*}$ & .2209 \\
Empatía situacional centrada en si mismo & .1522 & .1737 & .1070 \\
\hline
\end{tabular}

No obstante, consideramos oportuno mostrar los índices de correlación obtenidos entre el factor ansiedad o empatía centrada en sí mismo y la medida parcial de «Ayuda informada» (Tabla 2). Observamos que la empatía centrada en la víctima muestra una elevada asociación con esta medida. Era predecible cierta asociación si consideramos que ambas pruebas se pasaron inmediatamente después de relatar a los sujetos la situación de los niños de Colombia; no obstante, llama la atención la elevada asociación entre la empatía centrada en sí mismo o ansiedad y la ayuda informada en el grupo de las mujeres (.5079), mientras que entre los varones la correlación no es significativa.

Estos datos parecen sugerir que en las mujeres la empatía, incluso cuando es experimentada como ansiedad o malestar, predice una tendencia a la ayuda en la inmediatez de la situación, aunque este tipo de empatía centrada en sí mismo no predice un comportamiento prosocial-altruista general.

TABLA 2

Empatía situacional y medida de ayuda informada

\begin{tabular}{llll}
\hline & \multicolumn{3}{c}{ Ayuda informada } \\
& Muestra total & Mujeres & Varones \\
Empatía situac. centrada en la víctima & $.4176^{* *}$ & $.4409^{* *}$ & $.3170^{*}$ \\
Empatía situac. centrada en sí mismo & $.3740^{* *}$ & $.5079^{* *}$ & .1419 \\
\hline
\end{tabular}

Toma de perspectiva y conducta prosocial-altruista. La capacidad para situarse en la perspectiva del otro ha sido una de las variables consideradas por la literatura como importante predictor de la conducta prosocial. También en nuestro estudio previo se constató la importancia de esta capacidad de comprender los pensamientos y estados afectivos de los demás.

Pues bien, frente a nuestras predicciones al respecto, los índices de correlación obtenidos en este segundo estudio (Tabla 3) entre esta variable y las medidas de conducta prosocial-altruista no son significativos. Desde nuestro punto de vista, es posible que la escasa asociación se deba al carácter de la prueba, 
excesivamente sencilla para sujetos de 10-11 años y, por tanto, insuficientemente discriminativa.

\section{TABLA 3}

Toma de perspectiva y conducta prosocial altruista.

\begin{tabular}{lccc}
\hline & \multicolumn{3}{c}{ Conducta prosocial altruista } \\
Toma de perspectiva & .0722 &.-0872 & .2309 \\
\hline
\end{tabular}

No obstante, queremos llamar la atención sobre la diferencia entre ambos sexos, tanto en los índices de correlación con la conducta prosocial-altruista total, como con la ayuda informada (Tabla 4). Si en las mujeres la correlación es mínima, entre los varones alcanza un nivel si bien no significativo, tampoco despreciable (.2309 y .3983 , respectivamente).

\section{TABLA 4}

Toma de perspectiva y medida de ayuda informada

\begin{tabular}{lccc}
\hline & \multicolumn{3}{c}{ Ayuda informada } \\
& Muestra total & Mujeres & Varones \\
Toma de perspectiva & .2470 & .0107 & .3983 \\
\hline
\end{tabular}

A la vista de estos datos, se nos ocurre que, o bien la prueba fue más sencilla para el grupo de las niñas (quizás su proceso de desarrollo es más acelerado en este aspecto del conocimiento social), o bien los factores de naturaleza más conceptual sean más predictores de la ayuda inmediata en los varones que en las mujeres. Sobre esta cuestión volveremos más adelante.

Apego y conducta prosocial-altruista. Como se puso de manifiesto al presentar la oportunidad de estudiar esta variable, está suficientemente demostrado el papel de la historia afectiva en el desarrollo de la conducta prosocial. Asimismo, desde una perspectiva etológica, es muy coherente la vía por la cual se desarrolla la conducta altruista en el marco de la relaciones de apego (conducta altruista de los progenitores en la mayoría de las especies, intensos y continuados sentimientos afectivos que promueven el desarrollo de la empatía, modelos de aprendizaje de la conducta prosocial...). Sin embargo, nos ha sorprendido, y muy agradablemente, el gran poder predictivo de la seguridad del apego en el tema que nos ocupa. Como observamos en la Tabla 5, de entre todos los índices de correlación obtenidos en nuestro trabajo, el referido a la asociación entre apego y conducta prosocial-altruista es el más elevado, y especialmente el apego materno.

De nuevo podemos constatar diferencias ligadas al sexo. Es llamativa la fuerte relación entre el apego materno y la conducta prosocial-altruista en los varones.

Imagen del ser bumano y conducta prosocial altruista. A continuación se exponen las correlaciones obtenidas en los diferentes indicadores de la imagen 
del ser humano. Podemos comprobar (Tabla 6) que, para toda la muestra, se obtiene un índice de asociación muy elevado entre la concepción del ser humano como simpático y la variable criterio. Por otra parte, si bien el resto de los factores estudiados no alcanzan correlaciones significativas, la tendencia general se orienta en la dirección de nuestra hipótesis, con índices positivos y negativos relacionados con un concepto positivo y negativo, respectivamente, de la persona. Ello revierte en un nivel de asociación significativo entre la imagen general positiva del género humano y la conducta prosocial-altruista (.2070). Estos datos nos permiten afirmar cierto papel predictor de esta variable.

\section{TABLA 5}

Apego y conducta prosocial altruista.

\begin{tabular}{lccc} 
& \multicolumn{3}{c}{ Conducta prosocial altruista } \\
& Muestra total & Mujeres & Varones \\
Apego materno & $.3876^{* *}$ & $.3111^{*}$ & $.5217^{* *}$ \\
Apego paterno & $.2877^{* *}$ & $.2640^{*}$ & $.3803^{*}$ \\
\hline
\end{tabular}

Como hemos visto en apartados anteriores, también en este factor advertimos diferencias ligadas al sexo. Salvo en los aspectos referidos a la percepción de maldad y cariño, todos los factores muestran una diferencia superior a .10 a favor de los varones, que desde nuestro punto de vista podría apuntar de nuevo a una mayor determinación en éstos de los aspectos conceptuales, representativos, sobre la conducta prosocial-altruista.

TABLA 6

Imagen del ser bumano y conducta prosocial-altruista

\begin{tabular}{lccc}
\hline Imagen del ser humano & \multicolumn{3}{c}{ Conducta prosocial-altruista } \\
& Muestra total & Mujeres & Varones \\
\hline Bueno & .1660 & -0839 & $.3880^{*}$ \\
\hline Malo & -1614 & -1610 &.-1575 \\
\hline Cariñoso & .1495 & .1335 & .1810 \\
\hline Simpático & $.3076^{* *}$ & $.2527^{*}$ & $.4160^{*}$ \\
\hline Egoísta &.-1475 &.-0835 &.-2933 \\
\hline Triste & -0454 & -0781 & .2767 \\
\hline Imagen positiva total & $.2070^{*}$ & .1796 & .2716 \\
\hline
\end{tabular}

Disciplina y conducta prosocial-altruista. Los resultados obtenidos en nuestro estudio en relación con el tipo de disciplina utilizada de manera más frecuente por los padres y la conducta prosocial-altruista, en el total de la muestra, confirman nuestras predicciones (Tabla 7). Mientras la afirmación muestra una correlación negativa con la variable criterio, las prácticas disciplinarias orientadas 
a destacar las consecuencias de la acción del niño (prácticas inductivas) predicen mayores niveles de conducta prosocial-altruista, ya que si bien las correlaciones no son significativas al .01, podemos suponer que la razón se halla en el menor número de casos (no todos los padres enviaron el cuestionario).

Confirmando también la investigación precedente, parece claro en nuestros resultados que la retirada de amor, como técnica de control de la conducta infantil, en la muestra total no muestra relación con la variable criterio.

Sin embargo, el peso y la orientación de los índices de correlación varía cuando analizamos los resultados en mujeres y varones. Entre las niñas, si bien la inducción predice el comportamiento prosocial-altruista y la afirmación de poder correlaciona negativamente con el mismo, también la retirada de amor se asocia negativamente. Por el contrario, en los varones, ni la afirmación de poder ni la inducción se relacionan con la conducta prosocial, mientras que la retirada de amor parece predecirla en cierta medida.

\section{TABLA 7}

Disciplina y conducta prosocial-altruista

\begin{tabular}{lccc}
\hline Tipo de disciplina & \multicolumn{3}{c}{ Conducta prosocial altruista } \\
& Muestra total & Mujeres & Varones \\
\hline Afirmación de poder & -2149 & -3322 & .0258 \\
\hline Inducción & .2309 & .3065 & .0674 \\
\hline Retirada de amor & -0478 &.-2089 & .2326 \\
\hline
\end{tabular}

Conducta prosocial de los padres y conducta prosocial-altruista. Abordamos finalmente el último de los predictores considerados, confirmándose una vez más nuestras expectativas. Como podemos comprobar en la Tabla 8, la observación de modelos prosociales en el marco familiar correlaciona significativamente con la variable criterio, siendo el índice relacionado con el modelo materno más elevado en los varones que en las mujeres, lo que parece indicar una mayor influencia del modelo materno en aquéllos.

\section{TABLA 8}

Conducta prosocial de los padres y conducta prosocial-altruista

\begin{tabular}{lccc}
\hline Conducta prosocial padres & \multicolumn{3}{c}{ Conducta prosocial altruista } \\
& Muestra total & Mujeres & Varones \\
\hline C.P. madre & $.2187^{*}$ & .1690 & .3154 \\
\hline C.P. padre & $.2369^{*}$ & .2413 & .2411 \\
\hline C.P. padre y madre & $.2360^{*}$ & .2190 & .2763 \\
\hline
\end{tabular}




\section{Análisis discriminante}

Pasemos ahora a los resultados que mostró el análisis discriminante realizado para probar la potencia discriminativa del conjunto de variables predictoras de la conducta altruista, en definitiva, cuáles de ellas distinguen y caracterizan a los grupos seleccionados como muy altruistas y no altruistas (Hipótesis 2).

Las variables elegidas como predictoras en dicho análisis fueron las siguientes:

- Empatía como rasgo-disposicional.

- Imagen del ser humano.

- Empatía situacional centrada en sí mismo.

- Empatía situacional centrada en la víctima.

- Apego.

- Disciplina inductiva.

- Disciplina asertiva.

- Disciplina retirada de amor.

- Modelo prosocial padres.

En la variable «conducta prosocial-altruista» se establecieron tres categorías: «altruistas», «no altruistas» e «intermedios», utilizándose para el análisis únicamente las dos categorías extremas.

El índice Lambda de Wilks de la función discriminante fue de .37, con un Chi-cuadrado de 23.35 y una significación de .0055 . La correlación de la función con la pertenencia a los grupos altruista-no altruista es de .7936 .

La capacidad predictiva de la función puede observarse asimismo en el siguiente cuadro, que cruza la asignación a los grupos realizada a partir de la función discriminante con la pertenencia real a los dos grupos.

CuAdro II

Capacidad predictiva de la función discriminante.

\begin{tabular}{|lcccc|}
\hline \multicolumn{4}{c}{ PRONOSTICO PERTENENCIA GRUPO } \\
& & N. ${ }^{\circ}$ Casos & Altruistas & No altruistas \\
Perten. & Altruistas & 16 & 15 & 1 \\
Real & & & $93,8 \%$ & $6,3 \%$ \\
& No altruistas & 14 & 2 & 12 \\
& & & $14,3 \%$ & $85,7 \%$ \\
& Porcentaje casos bien clasificados por la función $=90,00 \%$ & \\
\hline
\end{tabular}

Por término medio el porcentaje de casos correctamente clasificados por la función es del $90 \%$. Esto representa un porcentaje bastante más alto del que cabría esperar por azar. Por lo tanto, podemos decir que, efectivamente, el conjunto de variables consideradas por nosotros como relevantes tienen un considerable poder predictivo respecto a los niveles de conducta prosocial-altruista.

Para realizar un análisis más pormenorizado de la naturaleza de la función discriminante podemos recurrir a examinar las correlaciones entre cada variable y dicha función. 
TABLA 9

Correlaciones entre las variables y la funcion

\begin{tabular}{ll}
\hline Empatía disposicional & .4576 \\
\hline Apego & .3322 \\
\hline Empatía situacional centrada en la víctima & .2937 \\
\hline Disciplina inductiva & .1882 \\
\hline Empatía situacional centrada en sí mismo & .1092 \\
\hline Modelo prosocial padres & .0703 \\
\hline Disciplina retirada amor & .0462 \\
\hline Imagen positiva ser humano & .0318 \\
\hline Disciplina asertiva & .0172 \\
\hline
\end{tabular}

De acuerdo con estas correlaciones, la función discriminante podría ser concebida como una función de la empatía como rasgo, la seguridad del apego, la empatía situacional centrada en la víctima y la disciplina inductiva.

Los resultados del análisis discriminante corroboran en gran medida nuestras predicciones. Podemos decir que la función discriminante compuesta por las variables seleccionadas clasifica correctamente a los sujetos altruistas y no altruistas. Sin embargo, también observamos que factores como la afirmación de poder como técnica disciplinaria, la imagen positiva del ser humano, la disciplina retirada de amor, el modelo prosocial de los padres y la empatía situacional centrada en sí mismo, factores que sí habían mostrado una relación con la variable criterio en los análisis bivariados con toda la muestra, no son variables fuertes en la caracterización de los dos grupos de sujetos seleccionados.

Por el contrario, la empatía disposicional, que en las correlaciones obtenidas con toda la muestra no mostró un índice significativo de asociación con el comportamiento prosocial-altruista, es el factor más potente en la caracterización de los sujetos seleccionados como claramente altruistas.

\section{DISCUSION}

Recapitulemos brevemente las conclusiones que pueden extraerse a partir de nuestro estudio.

1. Tanto en niños como en niñas constatamos que a mayor tendencia a empatizar con la víctima en una situación de necesidad, se da un mayor nivel de comportamiento prosocial-altruista. En las mujeres, además, incluso la ansiedad propia ante el dolor ajeno (empatía centrada en sí mismo) predice intención de ayudar.

2. Tanto en niños como en niñas se da una clara relación entre la seguridad del apego y la conducta prosocial-altruista. La asociación es más elevada con la seguridad del apego relativa a la figura materna. Por otra parte, la capacidad predictora del apego en la conducta prosocial-altruista es todavía más fuerte en los varones, sobre todo la del apego materno.

3. Parece confirmarse la relación entre la imagen positiva del género huma- 
no y la conducta prosocial-altruista. El hecho de que esta asociación sea más clara en los varones sugiere que en éstos los aspectos conceptuales, representativos, tienen un mayor poder predictor en el comportamiento altruista. Esta interpretación parece reforzada también por los resultados relativos a la variable toma de perspectiva. Este factor cognitivo, referido a la capacidad de situarse en la perspectiva del otro, y que según la literatura predice una conducta prosocialaltruista, en el presente estudio sólo ha mostrado una asociación importante con el comportamiento altruista en los varones; la correlación entre ambos factores en el caso de las niñas es insignificante.

4. En cuanto a la influencia del tipo de disciplina parental en el tema que nos ocupa, la predicciones se confirman sólo en el grupo de las mujeres, en el que la inducción muestra una relación positiva con la conducta prosocial, y tanto la afirmación de poder como la retirada del amor como técnicas disciplinarias se asocian negativamente con el comportamiento prosocial-altruista. Por el contrario, entre los varones la conducta prosocial sólo parece ser predicha por la retirada del amor, mientras que tanto la afirmación de poder como la inducción muestran una capacidad predictora prácticamente nula.

Se nos ocurre que el hecho de que la predicción relativa al influjo de la inducción en los varones no se vea confirmada por nuestros datos podría deberse al hecho de que los varones presentaran puntuaciones en su mayoría muy bajas, y con poca variabilidad, en dicho predictor. Existen estudios que confirman un menor uso de esta técnica disciplinaria con los varones que con las mujeres (Zussman, 1978; Hoffman, 1975b; Etxebarria, 1989).

5. La conducta prosocial-altruista muestra una importante relación con la conducta prosocial de los padres, siendo el modelo materno más influyente en los varones que en las mujeres. Este resultado corrobora los datos ya obtenidos en investigaciones anteriores sobre el tema (Hoffman, 1975c).

6. A la hora de intentar definir o caracterizar a los grupos más extremos en la variable que nos ocupa, los datos del análisis discriminante permiten proponer que los niños más altruistas son aquellos que disponen de un nivel elevado de empatía disposicional o empatía rasgo, los más seguros en la relación de apego con sus progenitores, aquellos que empatizan en situaciones de necesidad y cuyos padres utilizan como técnica disciplinaria más frecuente la inducción.

Esta conclusión corrobora básicamente nuestras predicciones. No obstante, debemos llamar la atención sobre un resultado aparentemente inconsistente. Nosotros habíamos predicho que la empatía disposicional mostraría una correlación significativa con la conducta prosocial-altruista. Pues bien, como se apuntó anteriormente, en los análisis bivariados la empatía disposicional no se asoció de manera significativa con el comportamiento prosocial-altruista. Sin embargo, nos encontramos que la empatía disposicional es la variable más fuerte a la hora de definir la función discriminante. Puede que la explicación de estos datos resida precisamente en que la empatía disposicional es un rasgo de personalidad que no tiene una distribución normal en la muestra, de modo que mientras no covaría con la conducta altruista-prosocial en la población general, los sujetos que son claramente definidos como altruistas disponen de este rasgo de personalidad, de esta tendencia estable a percibir la realidad desde el punto de vista de la víctima, a empatizar con ella.

En definitiva, todos los factores estudiados aquí muestran su capacidad predictiva en el comportamiento prosocial-altruista, siendo los más importantes los siguientes: la empatía, tanto disposicional como situacional, y la seguridad del 
apego. Una concepción positiva del género humano y la inducción como técnica disciplinaria en el marco familiar son también predictores de marcado poder.

Para terminar, podemos concluir que si queremos educar niños capaces de actuar a favor de los demás cuando éstos lo necesitan, es importante proporcionarles un clima familiar de seguridad afectiva y no desperdiciar las numerosas ocasiones que la propia vida cotidiana ofrece para sensibilizarles hacia los sentimientos y las necesidades de los otros, reforzando y potenciando las propias tendencias naturales de los niños a empatizar con el dolor ajeno.

Asimismo, el conocimiento de los factores predictores del comportamiento prosocial-altruista abre interesantísimas posibilidades para la intervención en el ámbito escolar tendente al desarrollo en el niño de esta capacidad humana.

El objetivo de nuestro actual trabajo es elaborar, partiendo de los resultados de la investigación básica realizada, posibles orientaciones didácticas para dicha intervención educativa. A esta tarea, que requiere imaginación y esfuerzo, animamos a participar a todos aquellos que crean que merece la pena desarrollar la capacidad humana de comportarse de manera altruista y que crean también que la escuela puede y debe plantearse acometer este reto.

\section{Referencias}

Bandura, A. (1987). Pensamiento y acción. Barcelona: Martínez Roca.

BATSON, C. D., y COKE, J. S. (1981). Empathy: a source of altruistic motivation for helping? En J. P. Rushton y R. M. Sorrentino (Eds.). Altruism and helping behavior: social, personality and developmental perpectives, Nueva Jersey; L. E. A.

Batson. C. D.; Darley, J. M., y Coke, J. S. (1987). Altruismo y amabilidad humana: Determinantes internos y externos de la conducta de ayuda. En L. A. Pervin y M. Lewis (Eds.) Perspectives in International Psychology. Nueva York (trad. cast. UNED, 1985).

Chacon, F. (1986). Una aproximación al concepto psicosocial de altruismo. Boletín de Psicología, 11, 41-62.

EISENBERG, N. (Ed.) (1982). The development of prosocial behavior. Londres: Academic Press.

Etxebarria, I. (1989). Sentimientos de culpa y cambio de valores morales. Tesis Doctoral: Universidad del País Vasco.

Fuentes, M. J. (1989). Análisis evolutivo de la empatía y la ansiedad como variables mediadoras del comportamiento de ayuda. Infancia y Aprendizaje, 48, 65-78.

Fuentes, M. J.; A podaka, P.; Etxeberria. I.; Fuentes, M. J.; Ledesma, A. R.; López. F., y Or. TIz. M. J. (1990). Predictores afectivos y cognitivos de la conducta prosocial. En prensa.

Fultz,J.; Batson, C. D.; Fortenbach, V.; McCarthy, P., y Varney, L. (1986). Social evaluation and the empathy-altruism hypothesis. Joumal of Personality and Social Psybology, 50, 761-769.

Hoffman, M. L. (1970). Moral development. En P. H. Mussen (Ed.), Carmichael's manual of child psychology (Vol. 2). Nueva York: Wiley.

Hoffman, M. L. (1975). Developmental synthesis of affect and cognition and its implications for altruistic motivation. Developmental Psychology, 11, 607-622.

Hoffman, M. L. (1975b). Sex differences in moral internalization and values. Joumal of Personality and Social Psychology, 32, 720-729.

Hoffman, M. L. (1975c). Altruistic behavior and the parent-child relationship. Joumal of Personality and Social Psychology, 31, 937-943.

Hoffman, M. L. (1977). Moral internalization: Current theory and research. En L. Berkowitz (Ed.). Advances in Experimental Social Psychology, Vol. 10. Nueva York: Academic Press.

Hoffman, M. L. (1980). Adolescent morality in development perspective. En J. Adelson (Ed.). Handbook of adolescent psychology. Nueva York: Wiley Interscience.

Hoffman, M. L. (1981). The development of empathy. En J. P. Rushton y R. M. Sorrentino (Eds.). Altruism and helping behavior: social, personality and developmental perspectives. Nueva Jersey: L. E. A.

HoffmaN, M. L. (1982). Development of prosocial motivation: Empathy and guilt. En N. Eisenberg $(\mathrm{Ed}$.). The development of prosocial behavior. Nueva York: Academic Press. 
Hoffman, M. L. (1983). Empathy, guilt and social cognition. En W. F. Overton (Ed.). The relationship between social and cognitive development. Londres: L. E. A.

Hoffman, M., y Salzstein, H. D. (1967). Parent discipline and the child's moral development. Joumal of Personality and Social Psychology, 5, 45-47.

IANNOTTI. J. R. (1978). Effects of role-taking experiences on role-taking, empathy, altruism and aggresion. Developmental Psychology, 14, 119-124.

IANNOTTI.J. R. (1985). Naturalistic and structured assessments of prosocial behavior in preschool children: the influences of empathy and perspective taking. Developmental psycho$\log y, 21,46-55$.

KalliopusKA, M. (1980). Children's belping behavior. Personality factors and parental influences related to helping behavior. Helsinki: Academia Scientiarum Fennica.

Krebs, Dennis, Miller y Date, T. (1985). Altruism and aggression. En Lindzey y Aronson (Eds.) Handbook of social psychology. Nueva York: Random House.

LóPEZ, F.; SÁnCHEZ, E., y JiMÉnEZ, F. (1988). Egocentrismo espacial: concepto y procesos implicados. Infancia y Aprendizaje, 43, 97-122.

Macauly. J. L. (1970). Altruism and helping behavior. Nueva York: Academic Press.

Mehrabian, A., y Epstein. N. (1972). A measure of emotional empathy. Journal of Personality. 40, 525-533.

Staub, E. (1979). Positive social Behavior and morality. Vol. 1 y 2. Londres: Academic Press.

Staub, E.; Bar.TAL, D.; Karlowski, J., y Reykowski, J. (Eds.) (1984). Development and maintenance of prosocial behavior. Nueva York: Plenum Press.

Tol, M., y Batson. C. D. (1982). More evidence that empathy is a source of altruistic motivation. Journal of Personality and Social Psychology, 43, 281-292.

Underwood, B., y Moore, B. (1982). Perspective-taking and altruism. Psychological Bulletin, 9, 143-173.

ZAHN. W strategies. En N. Eisenberg (Ed.). The development of prosocial behavior Nueva York: Academic Press.

Zussman, J. U. (1978). Relationship of demographic factors to parental discipline techniques. Developmental Psychology, 14. 